\section{Translational Dentistry: The New Frontier in Dental Practice}

\section{Abstract}

We stand at the edge of a new frontier in health care. The Affordable Care Act-2010 has encouraged researchers and clinicians to work constructively and cooperatively toward maximizing convergent translational patient-centered, effectivenessfocused and evidence-based health care. While developing innovative molecular and cellular biology techniques to inform translational research, the field has validated the sophisticated comparative effectiveness research protocol in translational effectiveness aimed at defining and characterizing the best evidence base for translational convergent health care in general, and translational dental practice in particular. The salient principles and criteria for translational convergent biomedicine of the XXI century are discussed in this paper.

Keywords: Affordable Care Act 2010; Translational research; Translational effectiveness; Comparative effectiveness research; Evidence-based dental practice

\section{Francesco Chiappelli1,2,3, Van- dan R. Kasar1,2, Jeong Min Hwang $^{1,2}$ and Olivia S. Cajulis ${ }^{2,3}$

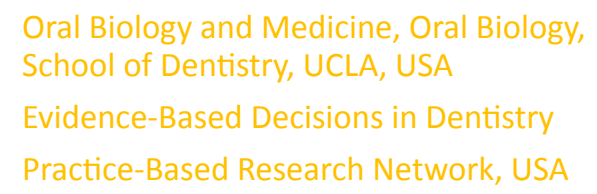

Francesco Chiappelli

fchiappelli@dentistry.ucla.edu

Ph.D., Dr. Endo (h.c.), Center for the Health Sciences, 63-090, UCLA-Dentistry, Los Angeles CA 90095-1668, USA.

Citation: Chiappelli F, Kasar VR, Hwang JM et al. Translational Dentistry: The New Frontier in Dental Practice. Transl Biomed. 2015, 6:4.

Received: December 03, 2015; Accepted: December 19, 2015; Published: December 23, 2015

\section{Introduction}

By signing the US Patient Protection and Affordable Care Act (PPACA, 2010; www.healthcare.gov/news/factsheets/overview. html), President Obama unquestionably changed health care in general and biomedicine specifically, throughout the U.S. To be clear, the new law is not perfect-future legislative actions will certainly converge to improve its breadth and its specifics. Nonetheless, PPACA has brought about a plethora of fundamental changes to guarantee the right to affordable, effectivenessfocused, patient-centered, and evidence-based health care for all its citizens. It signified a direct, timely, critical and significant application of the 2009 American Recovery and Reinvestment Act (ARRA, PL-111-5), which had allocated \$1.1B specifically for the development and the establishment of translational comparative effectiveness research (CER) and analysis in the health sciences [1], which the Institute of Medicine defined as" " the generation and synthesis of evidence that compares the benefits and harms of alternative methods to prevent, diagnose, treat, and monitor a clinical condition or to improve the delivery of care. The purpose of CER is to assist consumers, clinicians, purchasers, and policy makers to make informed decisions that will improve health care at both the individual and population levels..." [2]. (Institute of Medicine, 2009).
Embedded within its fundamental tenets, PPACA argues in favor of translational health care. The translational perspective in the health sciences consists of two independent yet intertwined facets. Initially, the National Institutes of Health (NIH) defined translational research as the transaction between the patient at the bedside/chairside and the fundamental pathobiology emerging from testing the patient's biopsies at the bench. Subsequently, the Agency for Healthcare Research Quality (AHRQ) outlined translational effectiveness as the process of obtaining and utilizing the best evidence base for certain treatment interventions in specific clinical settings, and of generating formative and summative evaluation in order to quantify the applications and the implications thereof for improved patient-centered health care. Translational science therefore represents two sides of the same coin: the concerted and complementary contribution of translational research and translational effectiveness in improving the health care process. This process begins from the initial patient-clinician encounter to the final prognostic follow-up, and includes all phases of diagnosis, delivery of care, treatment and monitoring in between $[1,3]$.

We have proposed elsewhere that a timely and critical 
question now can be paraphrased as follows: is it CER that drives translational effectiveness, meaning to say: is it the identification of the best evidence base that drives its utilization in specific clinical settings; or, on the contrary, is it translational effectiveness that drives the need for CER. This means that the information that is obtained by means of translational research and of translational effectiveness determines the search for the best evidence base and its utilization in clinical practice [3]. The question is as complex as it is timely and critical for translational health care in general and translational biomedicine in particular.

\section{Translational convergent health care}

It can be argued that the traditional divisions and segregations into fields and sub-fields, which have described Western biomedicine since the late XIX Century-e.g., psychiatry, anatomy, immunology, cardiology, dentistry, etc. -, while still prevalent today, are progressively being incorporated into a new and improved conceptualization of health care, which is referred to as "convergent". The intent of convergence in the health sciences is to join again the mind and the body, the mouth and the rest of the body, and Western biomedicine to medical traditions across multiple cultures (e.g., Ayruvedic Medicine, Traditional Korean Medicine).

In recognition of this timely evolution, NIH established the National Center for Advancing Translational Science (NCATS) with the purpose of defining and characterizing convergence in translational healthcare in biomedicine for the XXI Century. The Center aims at applying translational science in order to deliver new treatments and cures for disease to patients faster and more efficiently, and to disseminate the best evidence base more effectively to optimize collaborative approaches across medical traditions in the clinical enterprise. More importantly, however, the Center endeavors to ensure that patients are considered from the viewpoint of their physiological totality, meaning that biological variables must be considered in psychiatric patients, immunological diseases and disorders must not be isolated from the psychoneuroendocrine milieu that modulates all aspects of immune regulation, oral and dental conditions and diseases must be recognized for their significant impact on generalized health and disease, and psycho-physiopathology has a profound impact on oral pathology. We recently discussed these complex interactions in the specific context of the role of psychoneuroendocrineimmune interactions in the immune reconstitution inflammatory syndrome (IRIS) in patients with HIV/AIDS treated with antiretroviral therapy [4], as well as psychoneuroendocrine responses to stimuli (e.g., stress) that modulate immune systemic regulation and immune surveillance of the oral cavity [5]. In this context of convergence of dentistry into biomedicine, we also discussed the intertwined regulation among bone metabolism, and the psychoneuroendocrine and immune systems (i.e., osteopsychoneuroendocrine-immunology, [6] and its sequelae in such pathological conditions as HIV/AIDS [7].

Of particular current interests in the translational convergent health care that coalesces biodentistry with biomedicine are, for instance, problems of occlusion, which one might think to be primarily the domain of dentists, which can in fact significantly affect the temporomandibular joint, yielding significant biomarkers for translational research and producing remarkable head and neck neuralgias, with important central nervous system and neurologic consequences that inform and interest neurologists [8].

Additionally, orofacial cellulitis, a skin and soft tissue infection that presents as diffuse, erythematous swelling resulting from the spread of infectious bacteria across epidermal, dermal, and subcutaneous tissues, if left untreated, can spread throughout the stoma and neighboring tissues (e.g., superiorly, deep facial skeleton as a route to invade the central nervous system, or inferiorly, the laryngeal and pharyngeal areas thus provoking life-threatening compression of the airways), and alternatively lead to systemic sepsis. Orofacial cellulitis typically originates from complications in dental surgeries, root canal procedures or inadequate dental post-treatment care $[9,10]$.

\section{Implications and applications for dental practice}

In pursuit of translational science in dentistry, The American Dental Association defined evidence-based dentistry (EBD) as the approach to dental practice and to "...oral healthcare that requires the judicious integration of systematic assessments of clinically relevant scientific evidence, relating to the patient's oral and medical condition and history, with the dentist's clinical expertise and the patient's treatment needs and preferences...". From this viewpoint, evidence-based dental practice must, as does translational biomedicine, integrate the consensus of the best evidence base in a process of logic-based clinical decisionmaking that emphasizes both a focus on effectiveness-meaning to say, an emphasis on increased patient benefits and diminished risks at a lower cost-and on patient-centeredness [1-3,11].

Evidence-based dental practice is grounded, as translational biomedicine, on obtaining the best evidence base, and on incorporating into the treatment plan. In that regard, evidencebased dental practice distinguishes itself from dental practice based on the research evidence: whereas the latter utilizes the evidence in a few selected research reports, and therefore suffers from a bias of selection, the former is grounded on the best available research evidence that emerges from the systematic process of research synthesis, and reported as systematic reviews and meta-analyses. Evidence-based dental practice, as one essential component of translational healthcare, is a patientcentered, effectiveness-focused and evidence-based endeavor, which commences with the patient-clinician encounter at the diagnostic stage and culminates in the patient-clinician encounter during the entirety of the treatment prognostic and follow-up stages [1-3,11].

As a translational science of healthcare, evidence-based dental practice consists of two primary facets:

1. Translational research: emerging from the initial patientclinician encounter, biopsies and clinical tests are obtained to define and characterize the fundamental biological pathways that underlie the observed physiopathology.

2. Translational effectiveness: also emerging from the initial patient-clinician encounter, fundamental criteria are isolated by the clinician to discern the clinical literature 
that pertains specifically to the type of patient affliction $(P)$, the possible interventions $(I)$ and comparators $(C)$, the desired clinical outcome $(\mathrm{O})$, within the timeline under consideration (T) and the selected clinical settings (S). The crafted PICOTS question reveals the medical subject headings (MeSH) and keywords needed to uncover the pertinent clinical trials, observational studies and systematic reviews. Once obtained, the peer-reviewed evidence is assessed and evaluated for level (i.e., type of study) and quality (i.e., risk of bias). The consensus of the best evidence base results from a cogent analysis of the evidence ranked by level and quality.

For example, and in relation to the observations noted above, PICOTS questions could be as follows:

1. Temporomandibular joint disorder:

P-Adults of age 20 and older with temporomandibular joint disorder

I-Bite guards (dental splints)

C-Physical (musculo-skeletal) therapy

O-Reduced symptoms of temporomandibular joint disorder

$\mathrm{T}-1$ year follow up

S-Community dental practice

2. Cellulitis [10]:

P-Male and female patients of all ages diagnosed with cellulitis

l-erythromycin-based antibiotics administered orally

C-Penicillin-based antibiotics administered orally

O-elimination of threat to health from sepsis caused by cellulitis

T-2 weeks after antibiotic treatment

S-Hospital-dentistry setting

The tools and criteria for establishing the quality of the evidence are continuously being improved for reliability and validity. Case in point, the Ex-GRADE, a revision and expansion of the original Grading of Recommendations Assessment, Development, and Evaluation, which reliably establishes the assesses the published research's strength of the recommendation on the basis of the report study design, study quality, consistency, and directness $[1,3,9,11,12]$. The Risk of Bias instrument assesses the overall strength of evidence by measuring the risk of bias, derived from the lack of consistency, directness, and precision $[1,3,9,11,13]$. The Revised Assessment for Multiple Systematic Reviews provides (R-AMSTAR) establishes the methodological quality of systematic reviews by evaluating certain key criteria $[1,3,9,11,14]$. Related to the R-AMSTAR, the Preferred Reporting Items for Systematic reviews and Meta-Analyses (PRISMA) is a checklist of 27 items, which are regarded as indispensable factors of a transparent reporting of systematic reviews, that contain, or not, a metaanalysis $[1,3,11,15]$.

\section{Conclusion}

The New Frontier

It follows that we are at timely and critical cross-roads in dental practice, and more generally in translational biomedicine. We stand at the edge of a new frontier in health care: one ushered in by the PPACA-2010 five years ago, which encouraged researchers and clinicians to work constructively and cooperatively toward maximizing convergent translational patient-centered, effectiveness-focused and evidence-based health care. While developing sophisticated molecular and cellular biology techniques to inform translational research, the field has validated an increasing number of tools and instruments designed to evaluate the level and the quality of the research evidence. These comparative effectiveness research protocols are now available to define and characterize the best evidence base for translational convergent health care in general, and translational dentistry in particular. The principles and criteria for evidencebased medical and dental practice, are increasingly disseminated across the globe, as the foundations of translational convergent biomedicine in the XXI century.

\section{Acknowledgements}

The authors thank the Evidence-Based Decisions Active Groups of Stakeholders (EBD-AGS) of the EBD-Practice-Based Research Network (ebdpbrn.org), and the students and colleagues of the EBD Study Group, including and in particular Drs. André Bakkhordarian and Gary Demerjian. Funded in part by UCLA Senate grants and Fulbright Specialist grant (5077) to FC. 


\section{References}

Chiappelli F (2014) Fundamentals of Evidence-Based Health Care and Translational Science. Heidelberg, Springer-Verlag, Germany.

Institute of Medicine (2009) Initial National Priorities for Comparative Effectiveness Research. Washington, The National Academies Press DC.

Chiappelli F (2015) Methods, Fallacies and Implications of Comparative Effectiveness Research (CER) for Healthcare in the 21st Century. Chapter 1 In Comparative Effectiveness Research (CER): New Methods, Challenges and Health Implications Chiappelli $F$ (Edn.). Hauppauge, NOVA Publisher NY.

4 Chiappelli F (2015) Psychoneuroimmunology of Immune Reconstitution Inflammatory Syndrome (IRIS): The New Frontier in Translational Biomedicine. Translational Biomedicine 6: 11-14.

Chiappelli F, Bakhordarian A, Bach Q, Demerjian GG (2015) Translational Psychoneuroimmunology in Oral Biology \& Medicine. Forum on Immunopathological Diseases and Therapeutics In Press.

6 Chiappelli F (2011) Osteoimmunopathology: Evidence-Based Perspectives from Molecular Biology to Systems Biology. New York, Springer, NY.

7 Barkhordarian A, Ajaj R, Ramchandani MH, Demerjian G, Cayabyab $\mathrm{R}$, et al. (2011) Osteoimmunopathology in HIV/AIDS: A Translational Evidence-Based Perspective. Pathology Research International, Article ID 359242 epub.

8 Demerjian GG, Sims AB, Stack BC (2011) Proteomic signature of Temporomandibular Joint Disorders (TMD): Toward diagnostically predictive biomarkers. Bioinformation. 5: 282-284.
Bach Q, Kasar V, Chiappelli F (2015) Implications and applications of systematic reviews for evidence-based dentistry and comparative effectiveness research: A sample study on antibiotics for oro-facial cellulitis treatment. Dental Hypotheses 6: 134-140.

10 Boscolo-Rizzo P, Da Mosto MC (2009) Submandibular space infection: A potentially lethal infection. International Journal of Infectious Diseases 13: 327-333.

1 Chiappelli $F$ (2015) Involving community dentists in evidencebased dentistry: A hypothetical quest for the next frontier. Dental Hypotheses 6: 127-128.

2 Phi L, Ajaj R, Ramchandani MH, Brant XM, Oluwadara O, et al. (2010) Expanding the grading of recommendations assessment, development, and evaluation (Ex-GRADE) for evidence-based clinical recommendations: Validation study. Open Dentistry Journal 6: 3140 .

3 Barkhordarian A, Pellionisz P, Dousti M, Lam V, Gleason L, et al. (2013) Assessment of risk of bias in translational science. Journal of Translational Medicine 11: 184-186.

14 Kung J, Chiappelli F, Cajulis OO, Avezova R, Kossan G, et al. (2010) From systematic reviews to clinical recommendations for evidencebased health care: Validation of revised assessment of multiple systematic reviews (R-AMSTAR) for grading of clinical relevance. Open Dentistry Journal 4: 84-91.

15 Moher D, Liberati A, Tetzlaff J, Altman DG (2009) The PRISMA statement for reporting systematic reviews and meta-analyses of studies that evaluate healthcare interventions: explanation and elaboration. Journal of Clinical Epidemiology 62: 1006-1012. 